\title{
Trends in the hospital management of unstable angina
}

\author{
R F Heller, H Powell, R L O'Connell, K D’Este, L L Lim
}

\begin{abstract}
Study objective-To examine the hospital management of unstable angina (UAP) in 1996 and 1998, according to patient demographic variables and disease severity. Design-Medical record review.

Setting-37 hospitals across New South Wales, Australia, representative of the secondary and tertiary care hospitals in the State.

Participants-All patients (or a random sample of patients) with UAP admitted to these hospitals during five months in $\mathbf{1 9 9 6}$ and six months in 1998 (1872 and 1368 patients respectively).

Main results-In the two years between 1996 and 1998, there was an increase in the use of $\beta$ blockers and a corresponding decrease in the use of calcium channel blockers, as well as a decrease in the use of intravenous nitrates. Those aged 75 or more were roughly half as likely as those aged less than 65 to be prescribed heparin, aspirin and heparin, $\beta$ blockers, intravenous nitrates, and only one third as likely to be offered coronary angiography in hospital. They were one and a half times as likely to be prescribed calcium channel blockers compared with the youngest age group. A similar pattern was seen for gender, where men were more likely than women to be given aspirin, aspirin and heparin, and coronary angiography, and less likely to be given calcium channel blockers. Those with a past history were less likely, and those with more severe disease were more likely than others to be given most interventions.

Conclusions-In view of the low use of evidence-based management of UAP among women and the elderly, it would seem appropriate for disease management guidelines to target these groups. (F Epidemiol Community Health 2001;55:483-486)
\end{abstract}

The recommended management of unstable angina pectoris (UAP) has changed with the development of evidence as reflected in clinical practice guidelines. ${ }^{12}$ In November 1996, the Australian National Health and Medical Research Council released a set of guidelines that recommended the use of aspirin, intravenous or low molecular weight heparin, $\beta$ blockers and a rehabilitation programme-calcium channel blockers should be used only in certain clinical settings as should intravenous nitroglycerine, cardiac catheterisation and echocardiography. ${ }^{2}$ Despite many descriptions of variation in the management of acute myocardial infarction, ${ }^{3}$ there have been fewer reports of variation in the management of UAP although three papers have shown differences in management according to gender ${ }^{4}$ and age, ${ }^{45}$ and the presence of ongoing ischaemia. ${ }^{6}$ We took the opportunity to examine practice patterns among patients admitted to hospitals with UAP across the State of New South Wales, Australia in 1996 and 1998, in the context of a randomised controlled trial of an educational programme based on the new guidelines for UAP. ${ }^{7}$ We tested the hypothesis that there is variation in the use of guideline based management options over time and according to patient demographic variables as well as the severity of the disease.

\section{Methods}

Hospitals participated twice, in 1996 and in 1999 , in a stratified design to give adequate representation across hospital levels using the classification current in $1996 .^{8}$ All hospitals were selected from the nine principal referral, five major metropolitan and six major nonmetropolitan referral categories and a random sample taken from those in the district metropolitan (6 of 21), large district nonmetropolitan (6 of 12) and small district non-metropolitan (16 of 31) categories. Patients from principal referral, major metropolitan referral and district metropolitan hospitals were termed "metropolitan", and those from major non-metropolitan referral, large district non-metropolitan and small district non-metropolitan hospitals were termed "nonmetropolitan". Primary care hospitals in the categories of "community acute" and "ungrouped acute" were excluded because data collection was not cost effective given distances involved and the small number of eligible patients: these classes of hospitals account for only $3 \%$ of the total public hospital separations in NSW for UAP. Patients eligible for the baseline study in 1996 were those with an emergency admission to one of these hospitals between 1 February and 30 June 1996 with a principal discharge diagnosis coded according to ICD-9-CM 411.1 or 411.8. All patients admitted to major non-metropolitan referral, district metropolitan, large district nonmetropolitan and small district nonmetropolitan were included. Because of the larger numbers in the other two hospital groups, a random sample of 50 patients admitted to each principal referral hospital and 80 patients admitted to each major metropolitan referral hospital were included. (For the randomisation, the computer generated random numbers and sorted them, and the patients with lowest 50 numbers were selected.) A follow up survey was performed in 
Table 1 Characteristics of the patients according to year of survey. Figures are percentages unless otherwise stated

\begin{tabular}{lll}
\hline & 1996 & 1998 \\
\hline Hospitals (n) & 37 & 37 \\
Patients (n) & 1872 & 1368 \\
Metropolitan hospital & 60 & 58 \\
Age (y) & 36 & 37 \\
$\quad<65$ & 31 & 30 \\
$65-74$ & 33 & 33 \\
$75+$ & 59 & 60 \\
Male & 2 & 1 \\
Aboriginal or Torres Strait Islander & & \\
Insurance classification & 71 & 72 \\
$\quad$ Non-chargeable & 20 & 18 \\
Chargeable & 9 & 10 \\
Veterans Affairs chargeable & $4(2,6)$ & $4(2,6)$ \\
Median (quartiles) length of hospital stay (days) & & \\
Past history & 42 & 40 \\
$\quad$ AMI & 35 & 35 \\
Angina & 23 & 25 \\
$\quad$ No past history & 14 & 18 \\
Diabetes & 36 & 43 \\
Recurrent chest pain after admission & $0.7(12)$ & $1.5(20)$ \\
Developed acute myocardial infarction (n) & 38 & 38 \\
Heart failure & 59 & 64 \\
Severe & $0.5(9)$ & $0.5(7)$ \\
Died in hospital (n) & 17 & 18 \\
Transferred for angiography or revascularisation & & \\
Referred for follow up & 42 & 38 \\
GP & 19 & 15 \\
Physician & 33 & 32 \\
Cardiologist & & \\
& &
\end{tabular}

^Severity defined as presence of heart failure, recurrent chest pain or development of acute myocardial infarction.

1999, covering the period of admissions from 1 July to 31 December 1998, using a similar methodology and sampling techniques. All patients with a principal discharge diagnosis of UAP (ICD-10-CM-I20.0) were included if there were fewer than 50 patients over the study period in each hospital, and a random sample of 50 patients was taken if numbers were greater. Of the 48 hospitals that took part in the baseline survey, eight (all small district non-metropolitan) had fewer than five patients with acute cardiac chest pain admitted during the six month data collection period and were omitted from the intervention study. Hospital records were examined by nurses, who were given special training to minimise observer error. Data were collected on patient demographics, clinical signs and symptoms and hospital management. Data were collected on the drugs and procedures listed in the UAP practice guidelines. ${ }^{2}$ Checks were made on the accuracy of the discharge codes.

A randomised dontrolled trial was performed among the hospitals in this study and reported elsewhere. ${ }^{7}$ This involved education of clinical staff about the contents of the UAP guidelines as well as release of data on local practice patterns in relation to those in the

Table 2 Use of various inhospital drugs and procedures by year of survey (figures are percentages)

\begin{tabular}{llll}
\hline & 1996 & 1998 & $p$ Value \\
\hline Aspirin & 83 & 84 & 0.80 \\
IV/LMW heparin & 70 & 71 & 0.84 \\
Both aspirin and heparin & 62 & 63 & 0.95 \\
$\beta$ blocker & 48 & 54 & 0.027 \\
Calcium channel blocker & 54 & 44 & $<0.001$ \\
IV nitrates & 21 & 17 & 0.11 \\
Coronary angiography & 14 & 17 & 0.19 \\
Echocardiography & 13 & 12 & 0.71 \\
Rehabilitation & 26 & 23 & 0.40
\end{tabular}

IV, intravenous; LMW, low molecular weight. other hospitals surveyed. Hospitals in the control group were offered a similar intervention, without the release of data from the baseline survey, but held later so that any impact would occur after the time of hospital admission for the patients to be included in the follow up survey.

\section{STATISTICAL METHODS}

The survey commands in STATA, ${ }^{9}$ especially designed for use with complex survey data, were used. Hospital level (six groups) was specified as the strata and the individual hospitals were specified as the primary sampling units. Logistic regression analysis was performed to determine predictors of use of the drugs and procedures listed in the practice guidelines, while adjusting for potential confounders. Variables included in these models were: calendar year, intervention group, hospital type, age, sex, insurance classification, previous history of acute myocardial infarction/ angina or revascularisation, severity and diabetes. The severity variable was based on the presence of heart failure (clinical signs of ankle oedema, raised jugular venous pressure, pulmonary oedema or basal crepitations, radiological signs of pulmonary oedema or clinical diagnosis of heart failure), development of acute myocardial infarction or recurrent chest pain. Patients with any one of these conditions were defined as severe.

\section{Results}

Because of problems with record retrieval, one principal referral hospital was unable to participate in the follow up survey. One small district non-metropolitan hospital had no admissions with UAP over the six month period and two principal referral hospitals had merged with all cardiac admissions going to one of them. This left 37 hospitals who took part in the follow up-hospitals not featuring in the follow up were omitted from the baseline analysis to allow before-after analyses of the same hospitals.

Table 1 shows the characteristics of the patients surveyed during 1996 and 1998. These characteristics are similar in the two time periods and reflect a wide spread across metropolitan/non-metropolitan hospitals and patients ages, gender, and insurance classification. A majority of patients are classified as "severe" and have a previous history of cardiovascular disease, however inhospital mortality is low. Table 2 shows the raw percentages of use of the measured drugs and procedures that are similar in the two time periods with the exception of an increase in use of $\beta$ blockers and a decrease in the use of calcium channel blockers. Table 3 shows the results of multivariate analyses for each of the drugs, and table 4 shows the same for procedures. There were statistically significant changes between the two time periods in use of $\beta$ blockers, calcium channel blockers and intravenous nitrates - but no demonstrable change as a result of the randomised controlled trial of an educational intervention (as we have discussed in more 


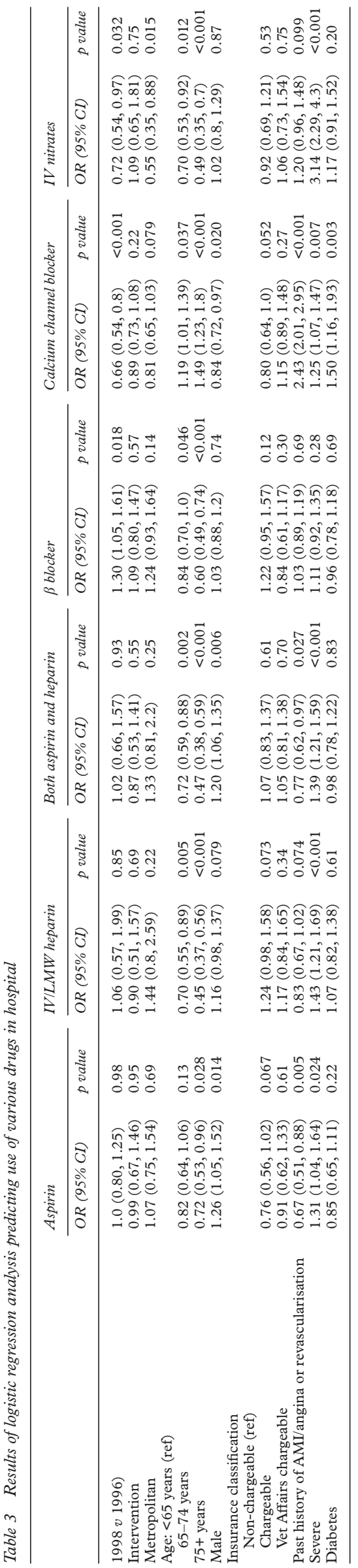
KEY POINTS
- Women and the elderly in hospital with unstable angina were less likely to be treated appropriately than men or younger patients.
- Patients with a past history and more severe disease were treated differently from those without these characteristics.
- Practice guidelines should make special mention of groups such as women and the elderly to increase the appropriateness of their care.
- Future research should examine the rela- tive impact of drugs and procedures on groups who may often be excluded from trials.

detail in a separate publication ${ }^{7}$ ). Older patients were significantly less likely to be given each of the interventions examined, with the exception of echocardiography and calcium channel blockers (whose use was significantly higher in the elderly). Women were significantly less likely to be given aspirin, aspirin and heparin, coronary angiography and more likely to be given calcium channel blockers. Those with a past history were significantly less likely to be given all interventions except calcium channel blockers (which they were more likely to be given) and nitrates and $\beta$ blockers (for which there were no statistically significant differences). Those with severe disease were significantly more likely to be given all interventions except for $\beta$ blockers; those with diabetes were more likely to be given calcium channel blockers. There were few differences according to insurance status, although nonchargeable patients were less likely to have an echocardiogram. The large differences between metropolitan and non-metropolitan hospitals in use of cardiac catheterisation and echocardiography have been commented on previously. ${ }^{10} 11$

\section{Discussion}

We have demonstrated large and statistically significant variations in the management of UAP in the two years between 1996 and 1998.

Guidelines for the management of unstable angina were released in Australia in November 1996, shortly after the first of our two surveys. ${ }^{2}$ In view of the lack of an effect following a special educational intervention based on these guidelines, it is unlikely that their release was responsible for the time trends we have shown. The most striking differences in management patterns are by age and sex. Those aged 75 or more are roughly half as likely as those aged less than 65 to be prescribed heparin, aspirin and heparin, $\beta$ blockers, intravenous nitrates, and only one third as likely to be offered coronary angiography in hospital. They are one and a half times as likely to be prescribed calcium channel blockers compared with the youngest age group. Patterns for those aged from 65 to 74 years are in between those in the oldest and youngest age groups. The guidelines to the management of UAP in Australia, ${ }^{2}$ 
Table 4 Results of logistic regression analysis predicting use of various procedures in hospital

\begin{tabular}{|c|c|c|c|c|c|c|}
\hline & \multicolumn{2}{|l|}{ Coronary angiography } & \multicolumn{2}{|l|}{ Echocardiography } & \multicolumn{2}{|l|}{ Rehabilitation } \\
\hline & OR $(95 \% C I)$ & $p$ value & OR $(95 \% C I)$ & $p$ value & OR $(95 \% C I)$ & $p$ value \\
\hline $1998 v 1996$ & $1.36(0.92,2.02)$ & 0.12 & $1.01(0.66,1.55)$ & 0.95 & $0.83(0.55,1.26)$ & 0.38 \\
\hline Intervention & $0.92(0.37,2.27)$ & 0.86 & $0.65(0.28,1.48)$ & 0.29 & $1.17(0.66,2.08)$ & 0.59 \\
\hline Metropolitan & $21.52(5.41,85.61)$ & $<0.001$ & $3.33(1.27,8.74)$ & 0.016 & $0.84(0.49,1.45)$ & 0.53 \\
\hline \multicolumn{7}{|l|}{ Age: $<65$ years (ref) } \\
\hline $65-74$ years & $0.80(0.62,1.03)$ & 0.084 & $1.32(0.98,1.79)$ & 0.070 & $0.96(0.74,1.25)$ & 0.77 \\
\hline $75+$ years & $0.35(0.21,0.59)$ & $<0.001$ & $1.30(0.94,1.8)$ & 0.11 & $0.69(0.50,0.94)$ & 0.022 \\
\hline Male & $1.55(1.09,2.21)$ & 0.016 & $1.04(0.82,1.31)$ & 0.76 & $1.03(0.82,1.29)$ & 0.80 \\
\hline \multicolumn{7}{|l|}{ Insurance classification } \\
\hline \multicolumn{7}{|l|}{ Non-chargeable (ref) } \\
\hline Chargeable & $1.23(0.95,1.6)$ & 0.11 & $1.47(0.99,2.19)$ & 0.057 & $0.98(0.67,1.43)$ & 0.92 \\
\hline Vet Affairs chargeable & $1.03(0.64,1.65)$ & 0.89 & $1.58(1.14,2.21)$ & 0.008 & $1.17(0.81,1.69)$ & 0.39 \\
\hline Past history of $\mathrm{AMI} /$ angina or revascularisation & $0.68(0.53,0.87)$ & 0.004 & $0.51(0.37,0.71)$ & $<0.001$ & $0.62(0.51,0.75)$ & $<0.001$ \\
\hline Severe & $1.56(1.12,2.16)$ & 0.010 & $1.36(1.13,1.64)$ & 0.002 & $1.31(1.08,1.59)$ & 0.007 \\
\hline Diabetes & $0.76(0.51,1.15)$ & 0.19 & $1.00(0.75,1.33)$ & 0.98 & $0.90(0.69,1.17)$ & 0.40 \\
\hline
\end{tabular}

which are similar to (and partly based upon) those in the USA from the AHCPR, ${ }^{1}$ suggest that aspirin, heparin and $\beta$ blockers are the main drugs of choice. Under some circumstances, calcium channel blockers may be used although $\beta$ blockers are preferred. If pain persists, intravenous nitrates are indicated, and a coronary angiogram (or an echocardiogram) might be performed. It would seem that those who are older are less likely to be given evidence based interventions than those who are younger. A similar pattern is seen for gender, where men are more likely than women to be given aspirin, aspirin and heparin, and coronary angiography, and less likely to be given calcium channel blockers.

Membership of a health fund, either through private insurance or Veterans Affairs insurance, was associated only with the use of echocardiography, hence in this setting insurance status had a negligible impact on management.

Those with a past history were less likely to be given most interventions except for intravenous nitrates, calcium channel blockers and $\beta$ blockers, and those with more severe disease were significantly more likely to be given all the interventions we report except $\beta$ blockers.

Others have reported differences in management of UAP, ${ }^{45}$ and other ischaemic heart disease syndromes ${ }^{12-15}$ by age and gender. The relative reluctance to use appropriate management for women and the elderly could be attributable to differences in doctors' perceptions of risk of either poor outcome or harm from the procedures among these groups or more seriously an indication of inadvertent neglect or discrimination. These results suggest that maybe it would be appropriate for disease management guidelines to make special mention of gender and age in an attempt to reduce the variation in management. The impact of disease severity and past history on management indicates the importance of including these variables in any analysis and the way in which patient characteristics can have a large influence on management.

We have shown that a number of patient characteristics have a large impact on the management of a common clinical problem-it is likely that much of this variation is not based on evidence. Future research should examine the relative impact of drugs and procedures on groups who may often otherwise be excluded from trials, namely women, the elderly, those with a past history and those with more severe disease.

Funding: the project was funded by the NSW Health Department and the National Health and Medical Research Department and the

Conflicts of interest: none.

1 Bruanwald E, Mark DB, Jones RH, et al. Unstable angina: diagnosis and management. Clinical practice guideline number 10. Rockville,MD: Agency for Health Care Policy and Research and the National Heart Lung and Blood Institute, Public Health Service, US Department of Health and Human Services, 1994:154.

2 Clinical practice guidelines: diagnosis and management of unstable angina. Canberra: National Health and Medical Research Council of Australia, November 1996.

3 Heller RF, O'Connell RL, Lim LLY, et al. Variation in stated management of acute myocardial infarction in five countries. Int 7 Cardiol 1999;68:63-7.

4 Scirica BM, Moliterno DJ, Every NR, et al. Differences between men and women in the management of unstable angina pectoris (The GUARANTEE Registry). Am $\mathcal{f}$ Cardiol 1999;84:1145-50.

5 Stone PH, Thompson B, Anderson HV, et al for the TIMI Stone PH, Thompson B, Anderson HV, et al for the TIMI
III Registry Study Group. Influence of race, age, and sex on management of unstable angina and non-Q-wave infarction: the TIMI III Registry. $7 A M A$ 1996;275:1104infarct.

6 Barakat K, Kennon S, Wilkinson P, et al. Selection bias in the management of unstable angina. I R Coll Physicians Lond 2000;34:179-84.

7 Heller RF, D'Este K, Lim LL-Y, et al. Randomised controlled trial to change the hospital management of unstable angina. Med F Aust 2001;174:217-21.

8 New South Wales Health. NSW Public Hospitals Comparison data 1994/95.

9 StataCorp. Stata statistical software: release 6.0. College Station, TX: Stata Corporation, 1999

10 Lim LLY, O'Connell R, Heller RF. Differences in management of heart attack patients between metropolitan and regional hospitals in the Hunter Region of Australia. Aust NZ F Public Health 1999;23:61-6.

11 Heller RF, O'Connell RL, D'Este K, et al. Differences in cardiac procedures among patients in metropolitan and non-metropolitan hospitals in NSW after acute myocardial infarction and angina. Aust 7 Rural Health 2000;8:310-17.

12 Naylor CD, Levinton CM. Sex-Related differences in coronary revascularisation practices: the perspective from a Canadian queue management project. Can Med Assoc $\mathcal{F}$ 1993;149:965-73.

13 McLaughlin TJ, Soumerai SB, Willison DJ, et al. Adherence to national guidelines for drug treatment of suspected acute myocardial infarction: evidence for undertreatment in women and the elderly. Arch Intern Med 1996;156:799805.

14 Shaw LJ, Miller DJ, Romeis JC, et al. Gender differences in the non-invasive evaluation and management of patients with suspected coronary artery disease. Ann Intern Med 1994;120:559-66.

15 Gan SC, Beaver SK, Houck PM, et al. Treatment of acute myocardial infarction and 30-day mortality among women and men. N Engl f Med 2000;343:8-15. 